\section{$\S 12$. Study of Electric Field Pulsation in Helical Plasmas}

Toda, S., Itoh, K.

The turbulence-driven transport and the formation mechanism of transport barriers are critical issues to realize improved confinement modes in toroidal plasmas. Main efforts have been focused on the understanding of the improved confinement modes (such as the High confinement mode (H-mode)). One thread of thoughts to explain the transport barriers is the structural transition in the profile of the radial electric field $E_{r}$ and the suppression of turbulence by its shear. The profile of $E_{r}$ strongly influences the confinement property in the toroidal plasmas. In helical plasmas, the neoclassical helical-ripple transport plays a dominant role in the process of forming the profile of $E_{r}$. The nonlinear relation between the neoclassical particle flux and the density and temperature gradients in the ambipolar condition induces the multiple solutions for $E_{r}$ at the local radial point in some cases. The bifurcation of $E_{r}$ in helical plasmas takes place and the resultant strong shear of $E_{r}$ was predicted to induce the transport barrier. At the state of the positive $E_{r}$ in the core region, the electron internal transport barrier (e-ITB) is observed when the Electron Cyclotron Resonance Heating (ECRH) is applied which is localized around the plasma center. The temporal evolution equation for the radial electric field is solved in helical plasmas. The cyclic phenomena in the temporal evolution of the radial electric field, namely the electric field pulsations, have been observed in the core region of Compact Helical System (CHS) and Large Helical Device (LHD). The temporally oscillating solutions of $E_{r}$ in the core region have not been shown yet. The transport model is constituted by the one-dimensional diffusion equations of the density, the electron and ion temperatures, and the radial electric field in helical plasmas. The results for the limit cycle of $E_{r}$, which oscillates between the two plasma states, are shown. The reduction of the transport due to the strong shear of $E_{r}$ is studied and the transport barrier with respect to the profile of the anomalous heat diffusivity is predicted. Repetitive change between the positive and negative states of $E_{r}$ is found to occur in the core region. A limit cycle of the radial electric field in helical plasmas is reproduced due to the multiple ambipolar $E_{r}$ under the condition where stationary sources are given for the particle and heat flux. The self-generated oscillation of the density and the temperature is found to take place. This result is attributed to the electric field pulsation observed in helical plasmas. The temporal transition between the positive $E_{r}$ and the negative $E_{r}$ occurs within much faster time scale than the confinement time.

The temporal evolution in the time interval $0.66 \mathrm{~s} \leq$ $\mathrm{t} \leq 0.70 \mathrm{~s}$ of $E_{r}$ in Fig. 1, $T_{e}, T_{i}$ and $n$ is studied. The solid line, the dotted-dashed line, the dotted line and the dashed line show the temporal evolutions of the physical quantities at $\rho=0.1, \rho=0.3, \rho=0.5$ and $\rho=1.0$, respectively. The state $A$ at $t=0.6721 \mathrm{~s}$ indicates the one at $\rho=0.3$ just before the transition from the negative $E_{r}$ to the positive $E_{r}$. Just after the transition from the state $A$, the state changes to the one $B$ at $t=0.6734 \mathrm{~s}$. From the state $B$, the plasma profile changes with the transport time scale and reaches the state $C$ at $t=0.6793 \mathrm{~s}$. Just after the state $C$, the transition from the positive $E_{r}$ to the negative $E_{r}$ takes place at $\rho=0.3$ and the state changes to the one $D$ at $t=0.6801 \mathrm{~s}$. From the state $D$, the plasma state changes to the state $A$ at $t=0.6833 \mathrm{~s}$ with the transport time scale. At the time $t=0.6833 \mathrm{~s}$, plasma parameters come back to the values at the state $A$, and the transition occurs again from the negative $E_{r}$ to the positive $E_{r}$. The temporal evolutions in the core region (at $\rho=0.1$, $\rho=0.3$ and $\rho=0.5$ ) clearly show the characteristic of the limit cycle. In the core region at $\rho=0.3$, the transitions occur from the negative $E_{r}$ to the positive $E_{r}$ at $t=0.6721 \mathrm{~s}$ and from the positive $E_{r}$ to the negative $E_{r}$ at $t=0.6801 \mathrm{~s}$. The time period of the limit cycle is about $10 \mathrm{~ms}$, which is determined by the typical transport time scale. The self-generated oscillation of the radial electric field is shown to have two time scales: a slow time scale of the transport time scale (about 10ms) and a fast time scale at the transition (about 1ms). The temporal change of the $E_{r}$ profile causes the temporal change of the radial profile of the neoclassical and anomalous diffusivities. Owing to the influence of $E_{r}$ on transport coefficients, the temporal evolution of the radial $T_{e}, T_{i}$ and $n$ profiles takes place as the limit cycle in the core region. The parameter region is shown for the self-generated oscillation: $1<\bar{T}_{e} / \bar{T}_{i}<2$ and $\bar{n} \approx 1 \times 10^{19} \mathrm{~m}^{-3}$, where the symbol $^{-}$represents the line-averaged quantity.

S. Toda and K. Itoh, 20th International Toki Conference (ITC20) Dec. 7-10 2010 P1-38.

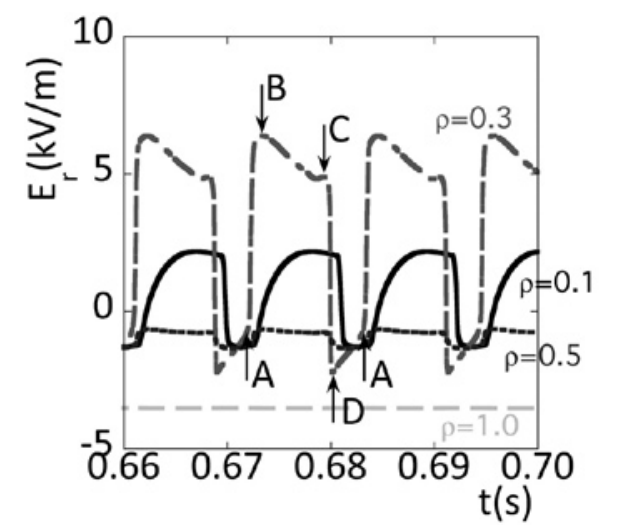

Fig. 1: Self-generated oscillation of the radial electric field 